\title{
22q11 DELETION SYNDROME: A SERVICE IMPROVEMENT PROJECT TO OPTIMISE CARE AND IMPROVE OUTCOMES
}

Dr Fiona Halton (fiona.halton@nhs.net), Dr Varsha Sadavarte (Varsha.Sadavarte@uhnm.nhs.uk): Community Paediatrics, Royal Stoke

University Hospital, Staffordshire, UK

22q11 deletion syndrome (DS) (also known as DiGeorge syndrome and Annually:

Velo-Cardio-Facial syndrome) is the most common chromosomal microdeletion syndrome in humans. It is estimated that 1 in 6000 children are affected, who can present with a range of different primary problems from congenital heart disease to communication difficulties.

In view of the fact that $22 q 11$ DS affects multiple body systems, children with 22 q11 DS can present to one or more of a number of different medical specialities. Often they can be under the care of multiple health care professionals, sometimes without MDT discussions or a coordinating consultant.

There are no national guidelines, and it can therefore be difficult to standardise care. We audited our paediatric population with 22q11 deletion syndrome at the Royal Stoke University Hospital, in an effort to improve the patient journey and standardise the way children are managed.

\section{MaxAppeal!}

MaxAppeal! is a UK registered charity supporting families affected by $22 q 11$ DS. The aim of their consensus document was "to compile a comprehensive and universally agreed lifelong care plan for people with 22q11DS within the framework of the NHS".

\section{Audit}

We audited all patients with a diagnosis of $22 q 11$ DS aged 16 or under on $1^{\text {st }}$ October 2017 against the recommendations set out by the MaxAppeal! 22q11DS consensus document (2013).

- Total of 18 patients identified

- Information available for only 15

- Age at diagnosis: antenatal -6 years

\section{At diagnosis:}

- Almost all had full blood count (FBC) and calcium checked, a cardiovascular system examination and $\mathrm{ECHO}$ as recommended.

- Most had renal scans and scoliosis examinations.

- Few had: lymphocyte subsets, immunoglobulins, thyroid function and post immunisation antibodies checked; audiology or eye examinations.

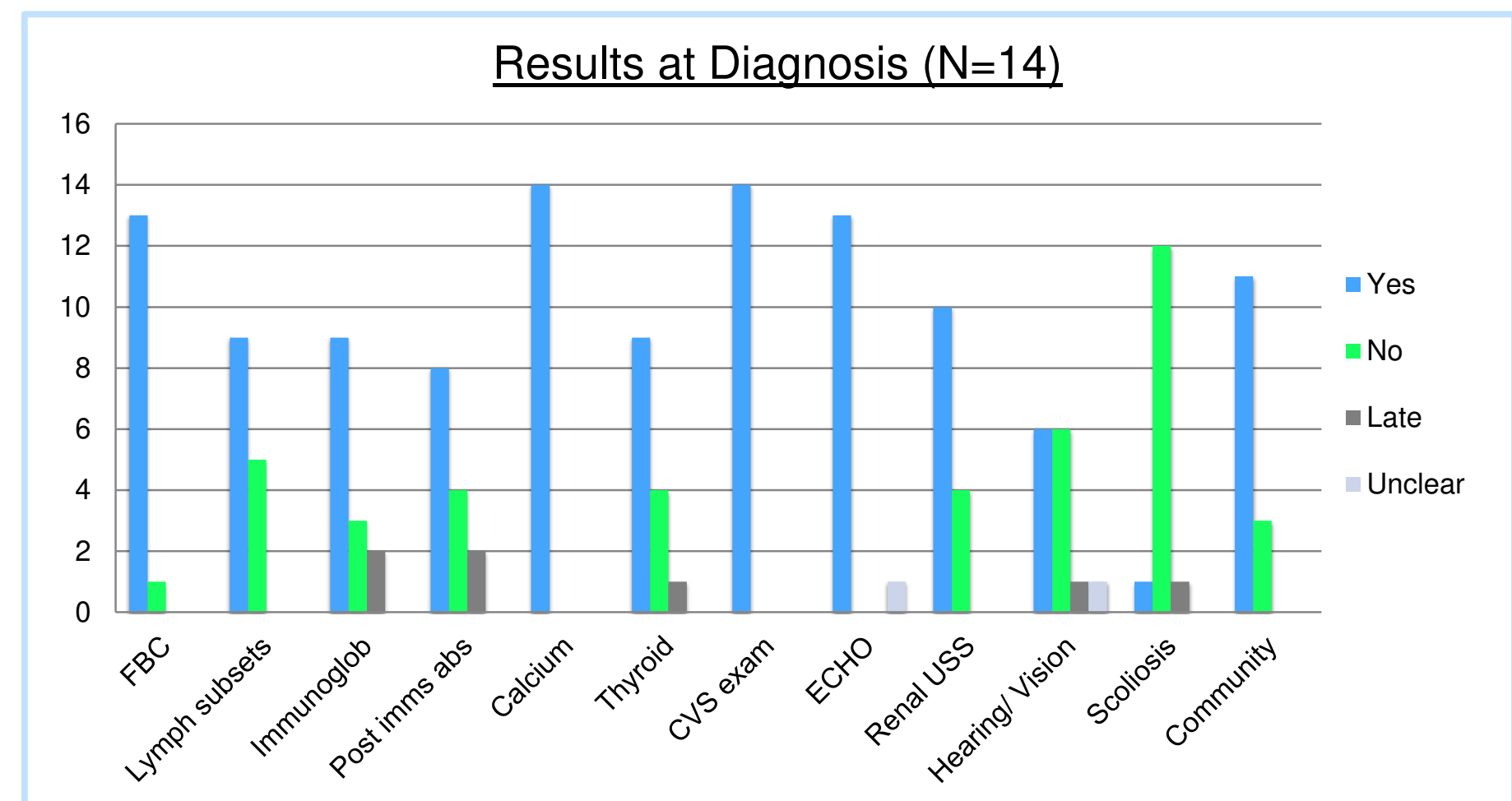

Patients were referred to allied healthcare professionals, and tertiary care specialists as needed, but not all were referred to community paediatrics.
- Only $27 \%$ had their FBC checked annually - this is important, as patients with 22q11 DS can present at any time with cytopenias.

- Few had: calcium levels or recommended thyroid screening; documented scoliosis examinations in early adolescence or advice given regarding regular dental care.

- None had documented absence of autoimmune conditions.

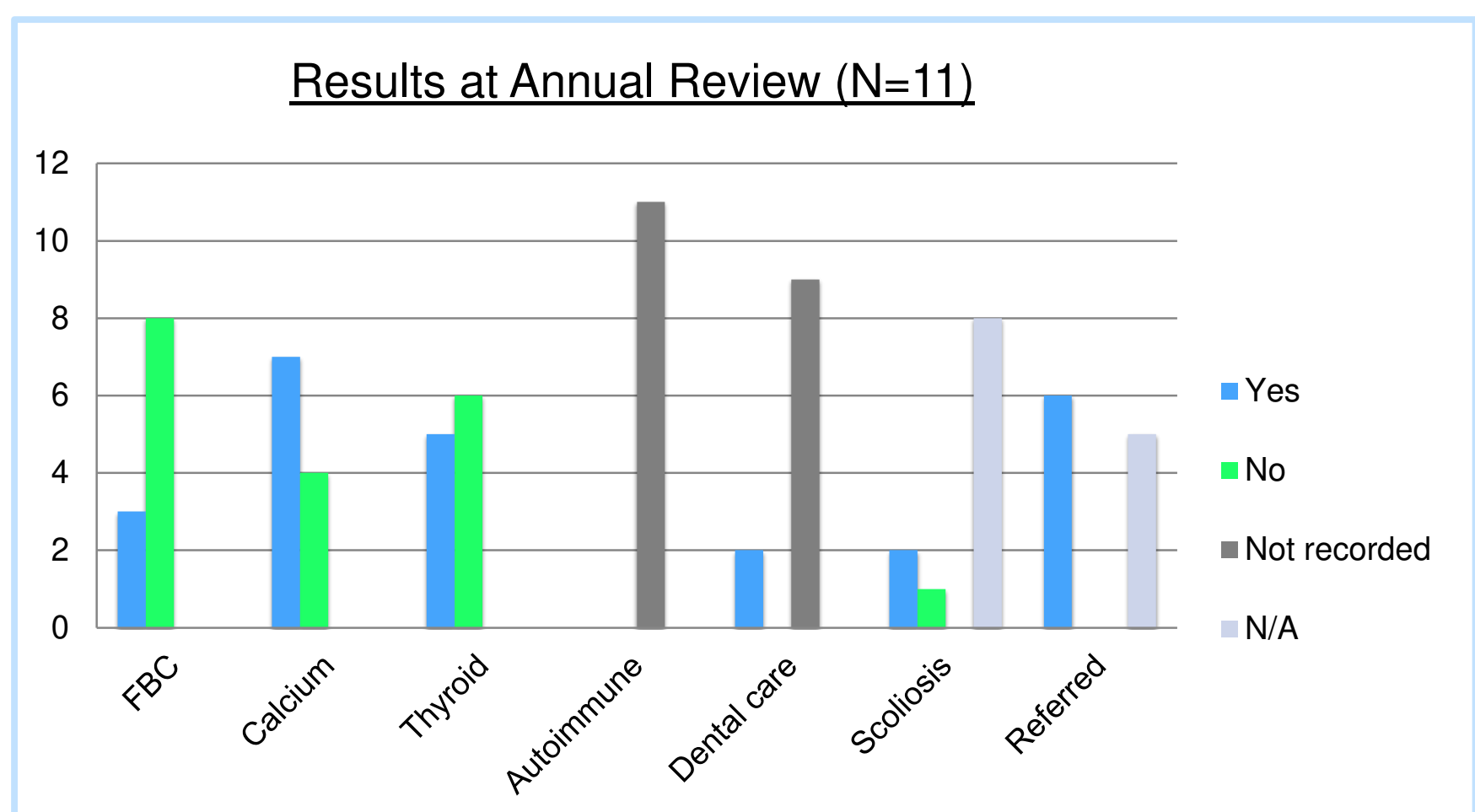

All patients were referred to allied healthcare professionals (physiotherapy, speech and language therapy) and tertiary services (immunology, cardiology) as required.

\section{Conclusion/ Service Improvement Project}

Our audit showed that many children were not having the recommended screening at diagnosis or annually. In order to facilitate standardised care, therefore, we have developed two checklists; to be used at diagnosis and annual review of any child with $22 q 11$ deletion syndrome.

As per the consensus guideline, all children will also be referred to a community paediatrician at diagnosis.

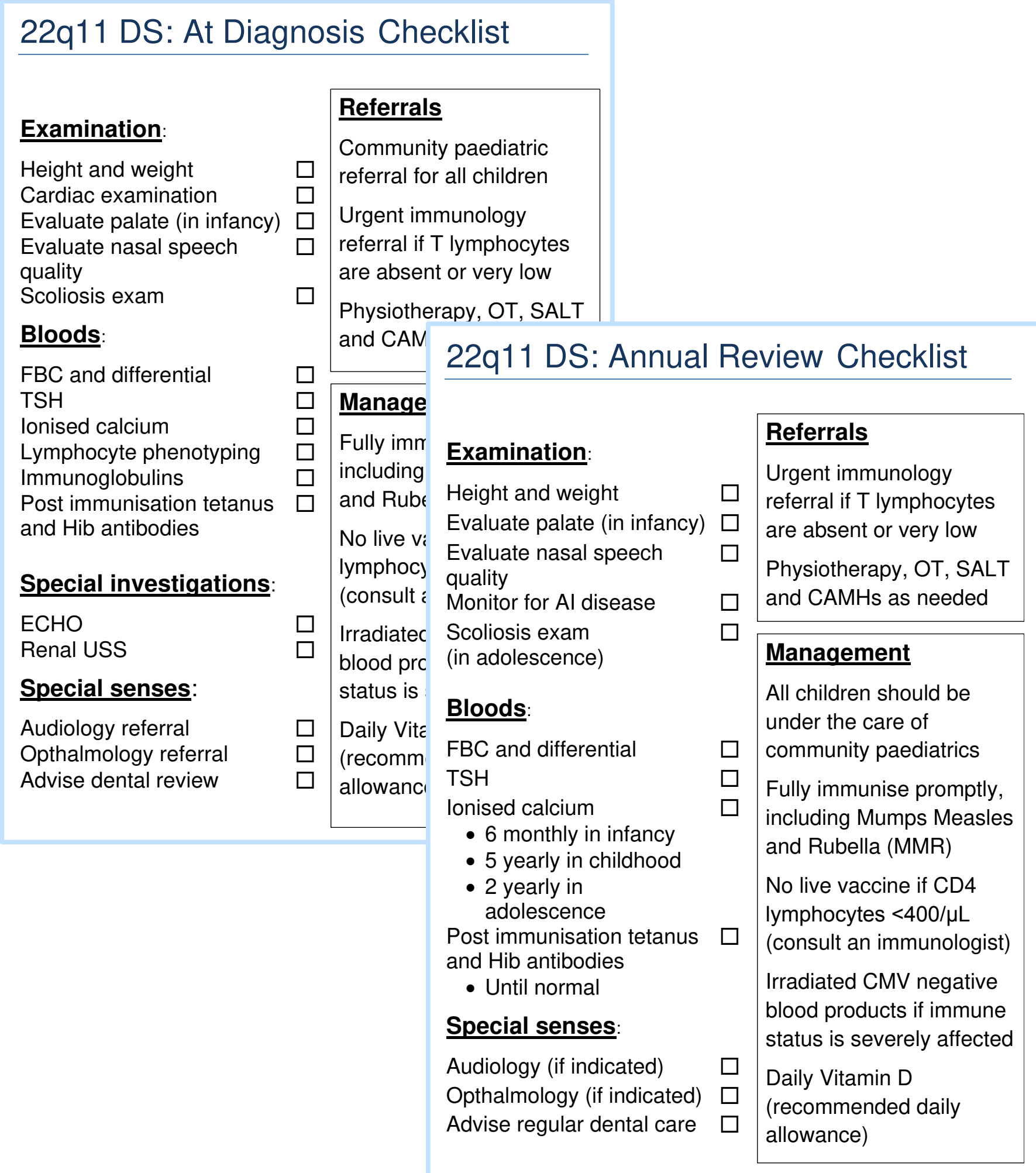

